\title{
Multiphoton Ionization Time-of-Flight Mass Spectrometry for the Detection of Bioactive Lignan
}

\author{
Tomohiro Uchimura, ${ }^{* \dagger}$ Goro Tokumoto, ${ }^{*}$ Onon Batnyam, $* *$ Chih-Wei Chou, $* * *$ and \\ Satoshi FuJITA**
}

\author{
*Department of Materials Science and Engineering, Graduate School of Engineering, University of Fukui, \\ 3-9-1 Bunkyo, Fukui 910-8507, Japan \\ **Department of Frontier Fiber Technology and Science, Graduate School of Engineering, University of Fukui, \\ 3-9-1 Bunkyo, Fukui 910-8507, Japan \\ ***Department of Cosmeceutic and Tsuzuki Institute for Traditional Medicine, China Medical University, \\ No. 91 Hsueh-Shih Road, Taichung, Taiwan 40402, R. O. C.
}

\begin{abstract}
Multiphoton ionization time-of-flight mass spectrometry (MPI-TOFMS) combined with a pulsed laser for sample vaporization was developed for the detection of a low-volatile compound in a solution. A solution containing Taiwanin A $((3 E, 4 E)$-3,4-bis(1,3-benzodioxol-5-ylmethylene)dihydro-2(3H)-furanone), which is a lignan that has an anticancer effect, was employed in the present study. Consequently, Taiwanin A could be detected by irradiating a laser pulse for vaporization to an inlet nozzle, rather than by heating. Therefore, the present method could be effective for detecting compounds with lower volatilities in a liquid sample.
\end{abstract}

Keywords MPI-TOFMS, lignan, Taiwanin A, anticancer drug

(Received August 19, 2015; Accepted September 15, 2015; Published February 10, 2016)

\section{Introduction}

Multiphoton ionization time-of-flight mass spectrometry (MPI-TOFMS) has spectroscopic selectivity and a robustness that prevents contamination, and is mainly an analytical means for measuring samples in their gas phase..$^{1-11}$ In the measurement of a liquid sample, a pulsed laser is often irradiated to a frit for vaporization. ${ }^{12,13}$ Recently, we reported on the use of vaporization and subsequent ionization techniques for measuring of a liquid sample without using a frit. ${ }^{14,15}$ Volatile compounds in aqueous solution are usually detected online. ${ }^{14}$ In that report, we irradiated a pulsed laser for vaporization to an inlet nozzle, and analyte molecules could be partially introduced in a pulsed form. Therefore, a signal enhancement was achieved, albeit a slight one.

In the present study, a direct analytical method of low-volatile compounds in a liquid sample was developed using MPITOFMS. Such an analytical technique would be useful for several applications. One example is an online analysis of pharmaceuticals in a liquid sample. As a model compound, we focused on lignans that have a common phenylpropane-skeleton as their fundamental unit. In particular, Taiwanin A (Fig. 1, $(3 E, 4 E)$-3,4-bis(1,3-benzodioxol-5-ylmethylene)dihydro-2(3H)furanone) can be extracted from Taiwania cryptomerioides Hayata, and possesses highly specific cytotoxic activities to cancer cells. ${ }^{16,17}$ Polymer nanofibers impregnated with Taiwanin $A$ are expected to be useful biomaterial structures with controlled

$\dagger$ To whom correspondence should be addressed.

E-mail: uchimura@matse.u-fukui.ac.jp drug release for various clinical uses. ${ }^{18}$ To confirm the behavior of drug release, an analytical system that can achieve online real-time monitoring of a liquid sample is required. In the present study, the use of MPI-TOFMS combined with laser vaporization was confirmed for the direct detection of Taiwanin $\mathrm{A}$ in a solution.

\section{Experimental}

Reagents and chemicals

About $1 \mathrm{~g}$ of purified Taiwanin A (b.p. $\left.201-202^{\circ} \mathrm{C}\right)^{19}$ was kindly provided by Y. H. Kuo (China Medical University, Taiwan), and was stored in a desiccator under shaded conditions before use. First, either a Taiwanin A standard solution dissolved in dimethyl sulfoxide was prepared, as shown in the measurement in Fig. 2, or dichloromethane was prepared, as shown in the

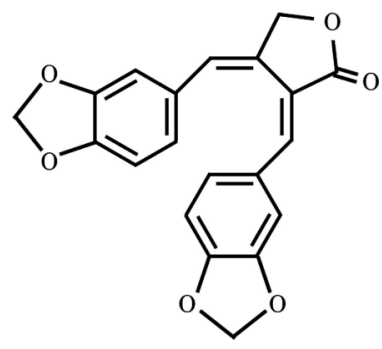

Fig. 1 Chemical structure of Taiwanin A. 


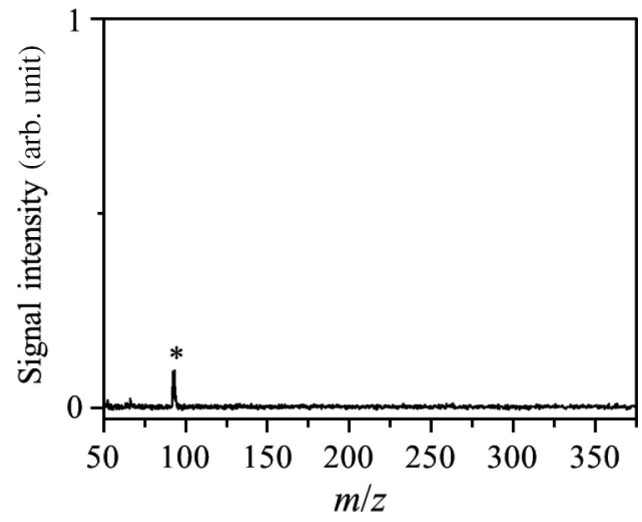

Fig. 2 Mass spectrum obtained by measuring a sample of a Taiwanin A solution sample by MPI-TOFMS. The peak for an internal standard (toluene) is marked with an asterisk $(*)$.

measurement in Fig. 3. The solution was diluted with dichloromethane for each experiment. Toluene, as an internal standard compound, was diluted with dichloromethane, and the solution was then added to the Taiwanin A solution.

\section{Apparatus}

The MPI-TOFMS used in the present study was described in a previous paper. ${ }^{14}$ Briefly, a linear-type TOFMS (drift length $60 \mathrm{~cm}$ ) was used, which is now commercially available (HikariGK, HGK-1, Fukuoka). A deactivated fused-silica capillary column (i.d., $25 \mu \mathrm{m}$, o.d., $375 \mu \mathrm{m}, c a .40 \mathrm{~cm}$ in length, GL Sciences) was connected to a TOFMS, and the capillary was directly inserted into a sample container. A heater was furnished on a flange of the TOFMS, and the inlet nozzle was indirectly heated to given temperatures, which are listed below.

The fourth-harmonic emission of a Nd:YAG laser $(266 \mathrm{~nm}$, $4 \mathrm{~ns}, 10 \mathrm{~Hz}$, Rayture Systems, GAIA II) was used for laser ionization. The laser pulse was focused via a lens $(\mathrm{f}=200 \mathrm{~mm})$, followed by the introduction of analyte molecules. The distance between an inlet nozzle and an ionization region was set at $1 \mathrm{~mm}$. For analyte vaporization from an inlet nozzle, the second-harmonic emission of a Nd:YAG laser $(532 \mathrm{~nm}, 5 \mathrm{~ns}$, $10 \mathrm{~Hz}$, Continuum, Minilite II) was used. The laser pulse was focused via a lens $(\mathrm{f}=200 \mathrm{~mm}$ ), which irradiated the tip of the capillary. The laser energy of the vaporization laser was $300 \mu \mathrm{J}$. The delay time for oscillation from the vaporization laser to the ionization laser was set to be $5 \mu \mathrm{s}$. The delay time was adjusted based on a previous report, ${ }^{14}$ where the delay time at which the strong signal intensity was observed was 7 to $12 \mu$ s when the distance between an inlet nozzle and an ionization region was set at $2 \mathrm{~mm}$. The speed of the molecules was calculated to be $c a .160-290 \mathrm{~m} / \mathrm{s}$, which is comparable to the present study ( $c a$. $200 \mathrm{~m} / \mathrm{s}$ ). The mass spectrum was recorded using either a digital oscilloscope (TDS5104, sampling rate $1.25 \mathrm{GS} / \mathrm{s}$, bandwidth $1 \mathrm{GHz}$, Tektronix) or a digitizer (AP240, sampling rate $1 \mathrm{GS} / \mathrm{s}$, bandwidth $1 \mathrm{GHz}$, Acqiris/Agilent Technologies). The mass spectra were averaged from measuring 100 or 600 single transients, which are shown in Figs. 2 and 3, respectively; they were shown after applying a second-order Butterworth lowpass filter with a cutoff frequency of 0.05 . The data were processed via a home-written program using graphical language (National Instruments, LabVIEW).

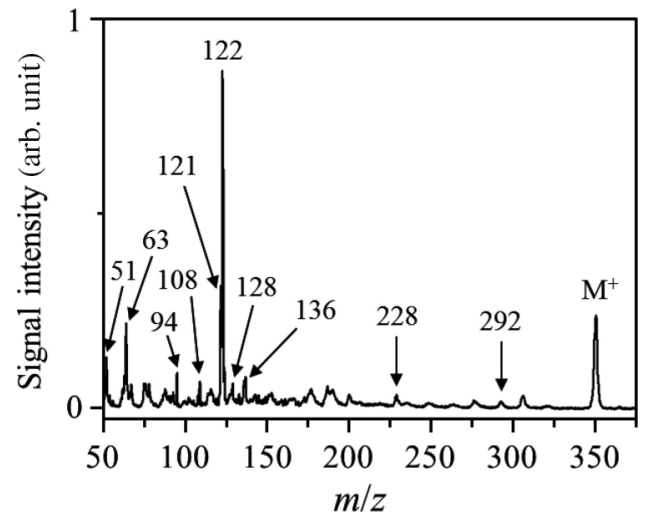

Fig. 3 Mass spectrum obtained by measuring a sample of a Taiwanin A solution by MPI-TOFMS combined with laser vaporization.

\section{Results and Discussion}

\section{Sample introduction by heating}

We first confirmed whether the less-volatile Taiwanin A in a sample solution could be vaporized and detected by heating the inlet nozzle, rather than by irradiating a pulsed laser for vaporization. The temperature of the inlet nozzle was successively increased to 220,250 , and $280^{\circ} \mathrm{C}$.

Figure 2 shows an example of the mass spectrum obtained by introducing $170 \mathrm{ng} / \mu \mathrm{L}$ of a Taiwanin A solution sample that contained $40 \mathrm{ng} / \mu \mathrm{L}$ of toluene. The temperature of the nozzle was $280^{\circ} \mathrm{C}$, and the energy of the ionization laser was $90 \mu \mathrm{J}$. As shown in Fig. 2, a peak arising from Taiwanin A was not detected, but toluene was clearly observed (marked with an asterisk). No peaks for Taiwanin A were observed by measurements at other temperatures. We determined that the temperatures of the inlet nozzle were insufficient to vaporize Taiwanin A and/or the ionization efficiency of Taiwanin A was rather poor. The temperature of the nozzle could not be raised to above $c a .300^{\circ} \mathrm{C}$ due to the specifications of the present apparatus. Therefore, a vaporization laser was applied to the sample introduction.

\section{Sample introduction using a pulsed laser for vaporization}

In the previous paper, we reported that the signal intensity for volatile 2,4-xylenol in a solution could be slightly increased by introducing a pulsed laser for vaporization, and discussed how further signal enhancement could be achieved when the laser vaporization technique was applied to less-volatile compounds. ${ }^{14}$ In the present study, the effect of a vaporization laser was studied for the detection of Taiwanin A.

Figure 3 shows the mass spectrum obtained by measuring a Taiwanin A solution $(10 \mathrm{ng} / \mu \mathrm{L})$ by MPI-TOFMS combined with laser vaporization. The nozzle temperature was set to $120^{\circ} \mathrm{C}$, and the energy of the ionization laser was $20 \mu \mathrm{J}$. As shown in Fig. 3, by using a vaporization laser, a molecular ion peak $(\mathrm{m} / \mathrm{z}, 350)$ and several fragment ion peaks of Taiwanin A were first detected by MPI-TOFMS. The detection limit, which was defined as the concentration providing a signal-to-noise ratio of 3, was $0.3 \mathrm{ng} / \mu \mathrm{L}$ calculated by using the molecular ion peak. The base peak was observed at $\mathrm{m} / \mathrm{z}, 122$ under the present conditions. The chemical structure of the species is unknown, but the 1,2-methylenedioxybenzene cation is a possible candidate due to the presence of an intense peak at $\mathrm{m} / \mathrm{z} 121$ (probably arising from the methylenedioxyphenyl cation). Therefore, efficient sample introduction was achieved for a 
less-volatile compound when using a vaporization laser. In future studies, by optimizing the experimental conditions, such as the energy of the vaporization laser, the wavelength of the ionization laser, and the temperature of the nozzle, a more sensitive analysis of many of the variants of bioactive lignans, including Taiwanin A, in a liquid will be accomplished.

\section{Conclusions}

In the present study, MPI-TOFMS combined with a sample introduction based on laser vapoprization was applied to the direct measurement of a less-volatile compound in a solution. As a result, Taiwanin A was detected using a vaporization laser, which suggested that this sample-introduction technique was efficient for the detection of less-volatile compounds. This system could be applied to online monitoring of the process for releasing Taiwanin A from impregnated nanofibers. Moreover, other anti-cancer drugs in solution could also be measured using the present method. The advantages of MPI-TOFMS, such as selectivity and robustness, should be effective for direct measurements of analytes in a mixture sample, such as a medium. We have reported the time-dependence of the uptake of anti-cancer drugs into a living cell in a medium by using fluorescence lifetime imaging microscopy (FLIM). ${ }^{20}$ By constructing a system that combines FLIM and MPI-TOFMS, the behavior of the uptake of drugs can be confirmed by FLIM, and drugs inside and outside the cells could be measured in succession by MPI-TOFMS, which would indeed be helpful in the development of anti-cancer drugs, as well as in evaluating the efficacy.

\section{Acknowledgements}

This work was supported by a research grant provided by the Kurita Water and Environment Foundation.

\section{References}

1. D. M. Lubman, R. Tembreull, and C. H. Sin, Anal. Chem.,
1985, 57, 1084.

2. J. Matsumoto, C.-H. Lin, and T. Imasaka, Anal. Chem., 1997, 69, 4524.

3. O. P. Haefliger and R. Zenobi, Anal. Chem., 1998, 70, 2660.

4. H. J. Heger, R. Zimmermann, R. Dorfner, M. Beckmann, H. Griebel, A. Kettrup, and U. Boesl, Anal. Chem., 1999, $71,46$.

5. H. Oser, K. Copic, M. J. Coggiola, G. W. Faris, and D. R. Crosley, Chemosphere, 2001, 43, 469.

6. L. Oudejans, A. Touati, and B. K. Gullett, Anal. Chem., 2004, 76, 2517.

7. K. Misawa, K. Tanaka, H. Yamada, Y. Goto, J. Matsumoto, Y. Yamato, S. Ishiuchi, M. Fujii, K. Tanaka, K. Endo, and S. Hayashi, Int. J. Engine Res., 2009, 10, 409.

8. Y. Hashiguchi, S. Zaitsu, and T. Imasaka, Anal. Bioanal. Chem., 2013, 405, 7053.

9. S. Sakurai and T. Uchimura, Anal. Sci., 2014, 30, 891.

10. A. Hamachi, T. Okuno, T. Imasaka, Y. Kida, and T. Imasaka, Anal. Chem., 2015, 87, 3027.

11. S. Otto, T. Streibel, S. Erdmann, M. Sklorz, D. Schulz-Bull, and R. Zimmermann, Anal. Chim. Acta, 2015, 855, 60.

12. J. Matsumoto, K. Kai, and T. Imasaka, Anal. Chem., 2003, 75,346 .

13. J. Matsumoto, K. Nishimura, T. Uchimura, and T. Imasaka, Anal. Chim. Acta, 2003, 484, 163.

14. G. Tokumoto, H. Saburi, S. Miyagawa, and T. Uchimura, Bunseki Kagaku, 2013, 62, 595.

15. H. Ishigami, Y. Tsuda, and T. Uchimura, Anal. Methods, 2014, 6, 5615.

16. S.-T. Chang, S.-Y. Wang, and Y.-H. Kuo, J. Wood. Sci., 2003, 49, 1 .

17. H.-J. Harn, H.-M. Chuang, L.-F. Chang, A. Huang, S.-T. Hsieh, S.-Z. Lin, C.-W. Chou, Y.-H. Kuo, and T.-W. Chiou, Fitoterapia, 2014, 99, 227.

18. O. Batnyam, H. Uematsu, C.-W. Chou, S. Suye, and S. Fujita, J. Biomater. Sci.-Polym. Ed., 2015, 26, 558.

19. S.-T. Chang, S.-Y. Wang, Y.-C. Su, S.-L. Huang, and Y.-H. Kuo, Holzforschung, 1999, 53, 142.

20. S. Kawanabe, Y. Araki, T. Uchimura, and T. Imasaka, Methods Appl. Fluoresc., 2015, 3, 025006. 